\title{
Development of selective immune tolerance towards the allogeneic fetus during pregnancy: Role of tryptophan catabolites (Review)
}

\author{
BAO TING ZHU \\ Department of Pharmacology, Toxicology and Therapeutics, School of Medicine, \\ University of Kansas Medical Center, Kansas City, KS 66160, USA
}

Received December 4, 2009; Accepted January 15, 2010

DOI: 10.3892/ijmm_00000411

\begin{abstract}
Studies in recent years have shown that the selective expression of indoleamine 2,3-dioxygenase (IDO) in certain cell populations may play an important role in mediating immunosuppression. Mechanistically, since IDO is a rate-limiting enzyme of the kynurenine pathway responsible for tryptophan catabolism, the prevailing explanation for its immunosuppressive action is based on the assumption that the presence of IDO in selected cell populations would consume local tryptophan and subsequently starve adjacent maternal T-cells of this essential amino acid. In this review, an alternative hypothesis is discussed, which suggests that IDO is mainly expressed in various types of antigen-presenting cells (such as placental syncytiotrophoblasts during pregnancy), and that its main function is to produce biologically-active tryptophan catabolites that will mediate immunosuppression. Mechanistically, because these tryptophan catabolites are concentrated in a microenvironment surrounding the IDOexpressing dendritic cells, they will selectively suppress the proliferation of a sub-population of T-cells that are activated by the allogeneic antigen-presenting cells and ultimately wipe out this T-cell sub-population. Evidence in support of this new mechanistic explanation is discussed. This hypothesis provides an alternative mechanistic explanation for the development of selective immune tolerance towards the allogeneic fetus during pregnancy. Moreover, it also offers insights into the functional role of certain tryptophan catabolites produced by cancer cells in evading the body's immune surveillance, as well as the potential usefulness of
\end{abstract}

Correspondence to: Dr B.T. Zhu, Department of Pharmacology, Toxicology and Therapeutics, University of Kansas Medical Center, MS-1018, Room KLSIC-4061, 2146 W. 39th Ave., Kansas City, KS 66160, USA

E-mail: btzhu@kumc.edu

Abbreviations: IDO, indoleamine 2,3-dioxygenase

Key words: indoleamine 2,3-dioxygenase, tryptophan catabolites, allograft tolerance tryptophan catabolites as pharmacological agents for the induction of selective long-term immune tolerance towards the allogeneic organ transplant.

\section{Contents \\ 1. Introduction \\ 2. An alternative hypothesis \\ 3. Review of the supporting evidence and discussion \\ 4. Concluding remarks}

\section{Introduction}

In today's medicine, the surgical techniques for organ transplantation are, in most cases, no longer an impeding problem. In contrast, the subsequent development of immune rejection against the organ transplant is, by far, the most significant obstacle to their long-term survival. Although many immune suppressants are available nowadays for clinical use, almost all of them have high levels of toxicity and adverse effects when they are used on a long-term basis (often for the rest of one's life). One of the most notable adverse effects of these immunosuppressive agents is their suppression of the body's immunity without selectivity, i.e., it will not only suppress the immune responses against the allograft, but it will also suppress the body's immunity against infection.

It is known that a woman's immune system during pregnancy normally does not reject the allogeneic fetus. Pregnancy represents a perfect model for the development of long-term selective immunotolerance towards an allogeneic fetus yet without reducing the overall disease-fighting immunity of the host. Understandably, seeking a better understanding of the mechanism of maternal immunotolerance of the fetus during pregnancy has been a subject of intense investigation for decades. Insights gained through these studies may offer strategies for developing novel therapeutic agents for the induction of selective, long-term immunotolerance towards the organ transplant.

Studies in recent years have shown that very high levels of indoleamine 2,3-dioxygenase (IDO) immunostaining were selectively detected in the syncytiotrophobasts of the 
placenta $(1,2)$. IDO, a rate-limiting enzyme of the kynurenine pathway, is largely responsible for the catabolism of tryptophan (3-6). It has been hypothesized that IDO expression in the placenta plays an important role in mediating the feto-maternal immune tolerance during pregnancy $(1,6-12)$. In support of this hypothesis, it was shown earlier that the pharmacological use of an IDO inhibitor (1-methyl-tryptophan) resulted in rapid, T-cellmediated rejection of allogeneic fetuses (1). However, this chemical inhibitor did not have a similar effect on geneticallyidentical (syngeneic) fetuses, thereby suggesting that its toxicity was not the cause for the fetal rejection observed (1). These studies provided strong support for the hypothesis that IDO is involved in the suppression of immune rejection during pregnancy.

Mechanistically, the current prevailing explanation for the immunosuppressive actions of IDO is based on the tryptophan depletion theory $(7,13)$, which assumed that the presence of IDO in certain types of cells would consume local tryptophan and subsequently starve adjacent maternal T-cells of this essential amino acid. Subsequently, this would lead to inhibition of local T-cell proliferation and ultimately immunosuppression. There were some experimental observations that appear to agree with the tryptophan depletion theory. For instance, studies have shown that in the absence of tryptophan, cultured T-cells were arrested at the mid-G1 point and were sensitized to undergo apoptosis (14). When T-cells were cultured together with macrophages or dendritic cells that were stimulated to express high levels of IDO, the proliferation of T-cells was inhibited $(1,8,14)$.

However, other possible alternatives to the typtophan depletion theory should also be considered for the following reasons: Firstly, in order for tryptophan depletion to be a viable mechanism for IDO-induced T-cell suppression in vivo, the concentrations of tryptophan most likely would have to be maintained at very low levels (probably $<0.5-1 \mu \mathrm{M}$ ) for a long period of time in the placental microenvironment. However, the plasma levels of tryptophan are usually in the range of 60-80 $\mu \mathrm{M}$; moreover, the rate of diffusion of tryptophan into the placenta likely is much faster than the rate of its local degradation. Consequently, it would seem rather difficult, if not impossible, for tryptophan depletion to work effectively as a viable mechanism for immunosuppression. Secondly, it is known that the placenta is an organ that supplies nutrients to the fetus and it would seem rather inconceivable if most or all of tryptophan (an essential amino acid) that circulates to the placenta is rapidly degraded by the placental IDO.

In this communication, an alternative mechanistic hypothesis is discussed, which provides an explanation for the selective development of T-cell-mediated immune tolerance towards the allogeneic fetus during pregnancy on the basis of IDO-mediated formation of certain immunosuppressive tryptophan catabolites. The mechanistic explanation tendered herein also offers insights into the functional role of tryptophan catabolites produced by cancer cells in mediating cancer metastasis. Lastly, the potential usefulness of tryptophan catabolites as pharmacological agents for the induction of selective immune tolerance towards the allogeneic organ transplant is also discussed.

\section{An alternative hypothesis}

It is known that very high levels of IDO are selectively expressed in placental syncytiotrophoblasts $(1,2,15)$, which constitute a unique group of fetal-derived cells that express allogeneic fetal antigens and are also in direct contact with the maternal bloodstream. When T-cells of the host immune system are circulated into the placenta, they will be in direct contact with the placental syncytiotrophoblasts. Earlier studies have shown that these syncytiotrophoblasts function as antigen-presenting cells (APCs) in the placenta. Because of the unique structural features of the placental sinuses and also the localization of syncytiotrophoblasts in these sinuses, these cells are highly favored to directly interact with blood lymphocytes circulated into the placenta. When the host T-cells are in contact with the fetal-derived syncytiotrophoblasts, these allogeneic APC cells will present the fetal antigens to the host T-cells, and a subpopulation of the maternal T-cells that can selectively recognize the fetal antigens will be activated, and subsequently these activated maternal T-cells will undergo rapid proliferation (presumably inside the placenta at the side of activation, i.e., near syncytiotrophoblasts) to develop cytotoxic T-cells as well as memory T-cells.

Theoretically, the cytotoxic T-cells could mount a T-cellmediated cytotoxic immune attack against all cells that express the fetal antigens. However, because the syncytiotrophoblasts express unusually high levels of IDO as well as other relevant enzymes along the kynurenine pathway, which will jointly catalyze the enzymatic conversion of tryptophan to a number of its catabolites. It is hypothesized that some of the biologicallyactive tryptophan catabolites with strong immune suppressive actions will be formed and they will contribute to the inhibition of the proliferation of T-cells through the induction of apoptotic cell death, and ultimately, effectively wipe out a sub-population of T-cells that are selectively activated by the allogeneic, fetal-derived syncytiotrophoblasts (with APC functions). Obviously, this sub-population of T-cells, if activated under abnormal conditions, would be responsible for the development of T-cell-mediated immune rejection against the allogeneic fetus.

In addition, it is hypothesized that the initiation of the humoral responses against fetal antigens will also be suppressed in a similar manner in the placenta. As such, both Th1 and Th2 responses against fetal antigens will be selectively inhibited in the placenta by tryptophan catabolites.

Lastly, it is also of note that since the bioactive tryptophan catabolites with a strong immune suppressive effect against T-cell proliferation are only formed locally in the placenta, they are not expected to have a significant effect on the proliferating T-cells outside the placenta. As such, while a selective immune tolerance towards the fetal antigens is effectively achieved in a pregnant woman's body, the immune system would still retain uncompromised functions against various forms of non-fetal antigens and infections.

\section{Review of the supporting evidence and discussion}

APC-activated T-cells are the target of action for tryptophan catabolites. As aforementioned, the current prevailing hypothesis that explains the immunosuppressive actions of IDO 
is based on the tryptophan depletion theory (13). However, there are also observations that suggest alternative possibilities. For instance, some studies reported that certain tryptophan catabolites formed along the IDO catabolism pathway could directly modulate the functions of immune cells $(10,11,18-21)$.

A recent in vivo study conducted at the author's laboratory examined the effect of 3-hydroxy-anthranilic acid (3-HAA), a tryptophan catabolite, which was injected into recipient animals together with donor-specific bone marrow-derived dendritic cells one week before the animals received the cardiac allograft (17). Under this experimental paradigm, it was expected that the donor bone marrow-derived dendritic cells would present donor-specific antigens to recipent's T-cells and activate the proliferation of alloreactive T-cells, whereas co-administration of 3-HAA would suppress the proliferation of alloreactive T-cells (likely the memory T-cells as well) that were selectively stimulated by the donor-specific bone marrowderived dendritic cells. The results showed that just a single i.v. injection of 3-HAA together with allogeneic bone marrowderived dendritic cells seven days prior to receiving the cardiac graft markedly prolonged its survival from seven to fifteen days.

In comparison, injection of 3-HAA alone seven days before receiving the cardiac graft had a very weak effect. To provide further evidence to support the proposed mechanistic explanation for the immunosuppressive effect of 3-HAA, additional experiments were conducted to examine the re-activation of donor-specific T-cells by allogeneic bone marrow-derived dendritic cells after the animals had received a single i.v. injection of 3-HAA + allogeneic bone marrowderived dendritic cells seven days earlier. The finding provided definitive support for the hypothesis that a sub-population of donor-specific T-cells is largely wiped out after a single injection of 3-HAA plus allogeneic bone marrow-derived dendritic cells. It is expected that multiple dosing of 3-HAA would increase the immunosuppressive effect.

Results from cellular and tissue distribution studies. It was shown recently that IDO was selectively expressed in various types of antigen-presenting cells (e.g., bone marrow-derived dendritic cells, macrophases, and placental syncytiotrophoblasts) (15). These observations also provide support for the proposed hypothesis that localized formation of certain tryptophan catabolites by IDO and other enzymes present in various antigen-presenting cells would serve as inhibitors of the T-cell-mediated immune responses against self-antigens, fetal antigens, or allogeneic antigens. Examples of the selective expression of IDO in cells with antigen-presenting functions as well as their implications are briefly discussed below.

Placenta and uterus. In the placenta, very high levels of IDO immunostaining were found to be selectively present in syncytiotrophobasts, a unique type of cells with antigenpresenting functions $(1,2,15)$. As explained above, the formation of immunosuppressive tryptophan catabolites in these cells would mediate selective immune tolerance towards the fetus. Here it is also worth noting that since the formation of adequate amounts of immunosuppressive tryptophan catabolites by the placental IDO does not require complete depletion of tryptophan, this would not pose a significant problem with respect to the necessity for the placenta to supply sufficient amount of essential nutrients (including tryptophan) for fetal growth.

In addition, IDO was also found to be expressed in the endometrium and myometrium of the uterus of sexually-mature female mice, and the staining was much stronger during the secretory phase (i.e., post-ovulation) $(15,22)$. It was suggested earlier that the presence of IDO in the uterine endometrium might help protect the female reproductive tract against ascending bacterial and parasitic infections by limiting the availability of tryptophan from exogenous sources. Whereas this possibility cannot be ruled out, it is somewhat puzzling that the uterine IDO levels are very low during the proliferative phase when the chances for bacterial infections are actually greater compared to the secretory phase due to the shedding of the endometrial tissue and the accompanying bleeding. An alternative explanation is that the selective expression of IDO in uterine endometrium during the secretary phase may be mostly associated with the suppression of the immune response against the allogeneic fertilized ova. In this instance, the IDOpositive uterine endometrial and myometrial cells would convert tryptophan to bioactive catabolites, which inhibit T-cell proliferation and exert immunoprotection of the allogeneic fetus(es).

GI tract. Another example is the GI tract, where a majority of cells in the Peyer's patches were found to be strongly stained for IDO (15). Peyer's patches are lymphoid aggregations within the lamina propria. IDO-positive cells in this lymphoid structure are thought to be closely related to its suggested role as modulators of the intestinal immune responses (15). Based on the localization and also morphological features of IDOpositive cells throughout the GI tract, IDO-positive cells are mostly antigen-presenting cells, and they are likely responsible for suppressing aberrant T-cell responses against food-borne cellular or noncellular immunogens, and ultimately to avoid aberrant autoimmune response against host tissues (as a result of cross reactivity).

Eye. In the mouse eye, positive IDO staining was detected in the corneal epithelium and the anterior chamber $(15,23)$. An earlier study also showed that while low levels of IDO mRNA and protein were detected in normal cornea, IDO expression was significantly increased following corneal transplantation (24). Following IDO cDNA transfer, murine corneal endothelial cells expressed functional IDO, which was effective in inhibiting allogeneic T-cell proliferation, and also prolonged the survival of corneal allografts (24). In addition, strong positive staining was detected in the outer limiting membrane, outer plexiform layer, and inner plexiform layer of the retina. The high IDO levels in these cells likely suggest that tryptophan catabolites may also play an important role in the development and maintenance of self-tolerance to immunologically privileged retinal antigens.

Cancer cells. It is also of note that many types of cancer cells express elevated levels of IDO. It is hypothesized that the same mechanism is also used by cancer cells to evade the T-cellmediated immune surveillance against immunogenic cancer cells, i.e., the IDO-expressing cancer cells will serve as APCs and suppress the proliferation of a sub-population of T-cells 
that can recognize the abnormal new self-antigens selectively expressed by the cancer cells.

Clinical usefulness of tryptophan catabolites as immunosuppressants. Based on the proposed hypothesis developed herein, it is apparent that some of the endogenously-present tryptophan catabolites, when used as pharmacological drugs in combination with donor's allogeneic dendritic cells, can selectively wipe out a subpopulation of T-cells that are activated by allogeneic antigens. Such a combined use of tryptophan catabolites with allogeneic dendritic cells would only need to be given for a very short period of time, such as starting a few days before receiving the organ transplant and lasting for a short period after the surgery. This strategy may be used to induce partial or complete long-term immune tolerance towards the allogeneic grafts. Theoretically, this strategy, if proven effective in humans, would be highly desirable because it may avoid the long-term use of immunosuppressive agents or help reduce the required doses of these immunosuppressants . In support of this strategy, it was recently shown that a single injection of 3-HAA, a tryptophan catabolite formed by the IDO metabolic pathway, could directly inhibit T-cell proliferation activated by the allogeneic bone marrowderived dendritic cells, and subsequently wipe out a specific sub-population of T-cells in vivo that would mediate allogeneic immune response against the allograft (17).

Another function of IDO - Role in the protection against infections. While IDO was found to be predominantly expressed in various antigen-presenting cells, there are also a few exceptions. One of the best-known cases in this regard is its expression in the caput of epididymis. In fact, the levels of IDO expression in these cells are the highest among all the tissues examined $(15,25)$. Based on the unusually high levels of IDO expressed in all apical epithelial cells (including their stereocilia), it is speculated that IDO may be able to completely deplete tryptophan inside the epididymal ducts of the caput. This depletion may serve the function of preventing bacterial and viral infections by suppressing the proliferation of bacteria and viruses. It is known that epididymitis is the most common cause of intrascrotal inflammation, most often caused by a secondary bacterial infection due to a variety of underlying conditions. Among them, urinary tract infections are the most common cause, and bacteria in the urethra can back-track through urinary and reproductive structures to epididymis. Based on this suggestion, the main function of the selective IDO expression in the caput of epididymis likely is to provide a segregated region that can prevent local bacterial or viral infections from spreading up along the seminiferous tubules where vitally-important spermatogenesis takes place.

It is of note that in prostate glands, cells with heavy IDO staining were detected almost exclusively in smooth muscle fibers and some of the epithelial cells of a dense connective tissue capsule that encloses the prostate glands. It is possible that the highly-localized strong IDO staining in prostate capasular cells may also be related to reducing local inflammation by suppressing the proliferation of pathogenic microorganisms (e.g., bacteria and viruses) through tryptophan depletion.

\section{Concluding remarks}

In this review, an emerging hypothesis is discussed, which suggests that the selective IDO expression in certain cell types may serve two main physiological functions: one is to deplete tryptophan in an enclosed microenvironment (such as inside the epididymal duct lumen) to prevent bacterial or viral infection, and the other function is to produce biologicallyactive tryptophan catabolites that will suppress T-cell-mediated immune responses against allogeneic fetal antigens as well as some of the self-antigens by suppressing a subpopulation of Tcells that are selectively activated by allogeneic antigenpresenting cells (such as placental syncytiotrophoblasts). Evidence in support of this new mechanistic explanation is discussed. This hypothesis provides a mechanistic explanation for the development of selective immune tolerance towards the allogeneic fetus during pregnancy. In addition, it also offers insights into the potential role of tryptophan catabolites produced by cancer cells in mediating cancer metastasis. Lastly, it is also suggested that tryptophan catabolites may be potentially useful as pharmacological agents for the induction of selective immune tolerance towards the allogeneic organ transplant.

\section{References}

1. Munn DH, Zhou M, Attwood JT, Bondarev I, Conway SJ, Marshall B, Brown C and Mellor AL: Prevention of allogeneic fetal rejection by tryptophan catabolism. Science 281: 1191-1193, 1998.

2. Mellor AL and Munn DH: Tryptophan catabolism prevents maternal T-cells from activating lethal anti-fetal immune responses. J Reprod Immunol 52: 5-13, 2001.

3. Hayaishi O: Properties and function of indoleamine 2,3dioxygenase. J Biochem 79: P13-P21, 2002.

4. Yoshida R, Nukiwa T, Watanabe Y, Fujiwara M, Hirata F and Hayashi O: Regulation of indoleamine 2,3-dioxygenase activity in the small intestine and the epididymis of mice. Arch Biochem Biophys 203: 343-351, 1980.

5. Takikawa O, Yoshida R, Kido R and Hayaishi O: Tryptophan degradation in mice initiated by indoleamine 2,3-dioxygenase. $\mathrm{J}$ Biol Chem 261: 3648-3653, 1986.

6. Belladonna ML, Puccetti P, Orabona C, Fallarino F, Vacca C, Volpi C, Gizzi S, Pallotta MT, Fioretti MC and Grohmann U: Immunosuppression via tryptophan catabolism: The role of kynurenine pathway enzymes. Transplantation 84 (Suppl 1): S17-S20, 2007.

7. Mellor AL and Munn DH: Tryptophan catabolism and T-cell tolerance: Immunosuppression by starvation? Immunol Today 20: 469-473, 1999.

8. Terness P, Bauer TM, Röse L, Duffer C, Watzlik A, Simon H and Opelz G: Inhibition of allogeneic $\mathrm{T}$ cell proliferation by indoleamine 2,3-dioxygenase-expressing dendritic cells: Mediation of suppression by tryptophan metabolites. J Exp Med 196: 447-457, 2002.

9. Mellor AL and Munn DH: IDO expression by dendritic cells: Tolerance and tryptophan catabolism. Nat Rev Immunol 4: 762-774, 2004.

10. Bauer TM, Jiga LP, Chuang JJ, Randazzo M, Opelz G and Terness P: Studying the immunosuppressive role of indoleamine 2,3-dioxygenase: Tryptophan metabolites suppress rat allogeneic T-cell responses in vitro and in vivo. Transpl Int 18: 95-100, 2005.

11. Radu CA, Bosch N, Bauer TM, Kleist C, Jiga L, Terness P, Opelz G, Gebhard MM, Germann G and Baumeister S: Immunosuppressive effect of tryptophan metabolites in composite tissue allotransplantation. Plast Reconstr Surg 119: 2023-2028, 2007.

12. Suzuki S, Tone S, Takikawa O, Kubo T, Kohno I and Minatogawa Y: Expression of indoleamine 2,3-dioxygenase and tryptophan 2,3-dioxygenase in early concepti. Biochem J 355: 425-429, 2001. 
13. Munn DH, Shafizadeh E, Attwood JT, Bondarev I, Pashine A and Mellor AL: Inhibition of T cell proliferation by macrophage tryptophan catabolism. J Exp Med 189: 1363-1372, 1999.

14. Lee GK, Park HJ, Macleod M, Chandler P, Munn DH and Mellor AL: Tryptophan deprivation sensitizes activated T cells to apoptosis prior to cell division. Immunology 107: 452-460, 2002.

15. Dai $X$ and Zhu BT: Indoleamine 2,3-dioxygenase tissue distribution and cellular localization in mice: Implications for its biological functions. J Histochem Cytochem 1: 1-12, 2010.

16. Munn DH, Sharma MD, Lee JR, Jhaver KG, Johnson TS Keskin DB, Marshall B, Chandler P, Antonia SJ, Burgess R, Slingluff CL Jr and Mellor AL: Potential regulatory function of human dendritic cells expressing indoleamine 2,3-dioxygenase. Science 297: 1867-1870, 2002.

17. Dai X and Zhu BT: Suppression of T-cell response and prolongation of allograft survival in a rat model by tryptophan catabolites. Eur J Pharmacol 606: 225-232, 2009.

18. Frumento G, Rotondo R, Tonetti M, Damonte G, Benatti U and Ferrara GB: Tryptophan-derived catabolites are responsible for inhibition of $\mathrm{T}$ and natural killer cell proliferation induced by indoleamine 2,3-dioxygenase. J Exp Med 196: 459-468, 2002.

19. Fallarino F, Grohmann U, Vacca C, Bianchi R, Orabona C, Spreca A, Fioretti MC and Puccetti P: T cell apoptosis by tryptophan catabolism. Cell Death Diff 9: 1069-1077, 2002.

20. Hackstein H and Thomson AW: Dendritic cells: Emerging pharmacological targets of immunosuppressive drugs. Nat Rev Immunol 4: 24-34, 2004.
21. Weber WP, Feder-Mengus C, Chiarugi A, Rosenthal R, Reschner A, Schumacher R, Zajac P, Misteli H, Frey DM, Oertli D, Heberer M and Spagnoli GC: Differential effects of the tryptophan metabolite 3-hydroxyanthranilic acid on the proliferation of human $\mathrm{CD}^{+} \mathrm{T}$ cells induced by TCR triggering or homeostatic cytokines. Eur J Immunol 36: 296-304, 2006.

22. Sedlmayr P, Blaschitz A, Wintersteiger R, Semlitsch M, Hammer A, MacKenzie CR, Walcher W, Reich O, Takikawa O and Dohr G: Localization of indoleamine 2,3-dioxygenase in human female reproductive organs and the placenta. Mol Human Reprod 8: 385-391, 2002.

23. Chiarugi A, Rapizzi E, Moroni F and Moroni F: The kynurenine metabolic pathway in the eye: studies on 3-hydroxykynurenine, a putative cataractogenic compound. FEBS Lett 453: 197-200, 1999.

24. Beutelspacher SC, Pillai R, Watson MP, Tan PH, Tsang J, McClure MO, George AJ and Larkin DF: Function of indoleamine 2,3-dioxygenase in corneal allograft rejection and prolongation of allograft survival by over-expression. Eur J Immunol 36: 690-700, 2006.

25. Britan A, Maffre V, Tone S and Drevet JR: Quantitative and spatial differences in the expression of tryptophan-metabolizing enzymes in mouse epididymis. Cell Tissue Res 324: 301-310, 2006. 